\title{
The Impact of National Surveillance for Liver Cancer: Results from Real- World Setting in Korea
}

\author{
Jin Won Kwon ${ }^{1,2}$, Ha Jin Tchoe ${ }^{1}$, Jayoun Lee ${ }^{1}$, Jae Kyung Suh ${ }^{1}$, Jeong-Hoon Lee ${ }^{1,3}$, and Sangjin Shin ${ }^{1}$ \\ ${ }^{1}$ Division of Healthcare Technology Assessment Research, National Evidence-based Healthcare Collaborating Agency, Seoul, ${ }^{2}$ College of \\ Pharmacy and Research Institute of Pharmaceutical Sciences, Kyungpook National University, Daegu, and ${ }^{3}$ Department of Internal Medicine \\ and Liver Research, Seoul National University College of Medicine, Seoul, Korea
}

Background/Aims: The National Liver Cancer Surveillance Program (NLCSP) was established in 2003 to reduce the socioeconomic burden imposed by liver cancer (LC). We aimed to investigate the effectiveness of the NLCSP in South Korea with respect to survival benefits and cost, after adjusting for various confounding factors. Methods: We used the National Health Insurance Service claims data linked with the NLCSP from 2004 to 2015. The Cox proportional hazard model and generalized linear model were used to determine the effects of the NLCSP on the early detection of LC, survival, and medical costs. Results: From 2006 to 2010, 66,632 patients (surveillance group: 10,527 and no surveillance group: $56,105)$ newly diagnosed with LC were included in the study. The odds of the early detection of LC was 1.82 (95\% confidence interval [Cl], 1.73 to 1.93) times higher among patients who participated in the NLCSP once within the 2-year period prior to the diagnosis of LC than among those who did not participate in the surveillance program. The mortality rate of patients who participated in the NLCSP was $22.0 \%$ lower (hazard ratio, $0.78 ; 95 \% \mathrm{Cl}, 0.76$ to 0.80 ) than that of those who did not participate. When compared with the group who did not participate in surveillance, the group who participated in the NLCSP had higher total medical costs; however, their cost per day was lower after adjustment during the follow-up period. Conclusions: This study highlights the survival benefit in patients who participated in the NLCSP and the need for continuous improvements of the NLCSP in South Korea. (Gut Liver 2020;14:108-116)

Key Words: Liver neoplasms; Early detection of cancer; Republic of Korea

\section{INTRODUCTION}

Primary liver cancer (LC) is one of the most common cancers in 2012, accounting for 9.1\% of all cancer worldwide and the second most common cause of death from cancer worldwide and same in South Korea. ${ }^{2}$ LC mortality rates mirror hepatocellular carcinoma (HCC) incidence rates, reflecting the poor survival of this cancer. ${ }^{3}$ HCC is the most common type of LC accounting for approximately $75 \%$ of all LC. ${ }^{4}$

HCC meets all the criteria established by the World Health Organization (WHO) for performing surveillance on those at risk for developing cancer. ${ }^{5}$ The rationale for conducting the HCC surveillance is that the regular screening of at-risk asymptomatic patients may detect tumors at an early stage when potentially curative treatment can be offered. Patients who were diagnosed with cirrhosis according to the National Veterans Administration clinical practice guidelines and underwent HCC surveillance 2 years prior to the diagnosis, had better health outcomes. Patients who underwent HCC surveillance are diagnosed at an early stage of HCC (Barcelona Clinic Liver Cancer stage 0/A $27.2 \%$ vs $11.6 \%$ ) and are likely to receive appropriate treatment as compared with the no surveillance group. ${ }^{6}$ Therefore most of LC surveillance program targets HCC detection.

The prevalence of hepatitis B virus and hepatitis $C$ virus infections in Asia, well known risk factors for HCC, is higher than Western counterparts. ${ }^{7,8}$ Therefore, surveillance systems for HCC are needed in Asia; in addition, Japan and South Korea have created the national program for HCC surveillance. Japan is one of the few countries that established the nationwide surveillance program since 1980, which covered the expense of the patient and used a more stringent guideline (more screening tests and

\footnotetext{
Correspondence to: Sangjin Shin

Division for Healthcare Technology Assessment, National Evidence-based Healthcare Collaborating Agency, 173 Toegye-ro, Jung-gu, Seoul 04554, Korea

Tel: +82-2-2174-2750, Fax: +82-2-747-4918, E-mail: jshin@neca.re.kr

Received on November 28, 2018. Revised on February 11, 2019. Accepted on February 12, 2019. Published online July $24,2019$.

pISSN 1976-2283 eISSN 2005-1212 https://doi.org/10.5009/gnl18522

Jin Won Kwon and Ha Jin Tchoe contributed equally to this work as first authors.

(a) This is an Open Access article distributed under the terms of the Creative Commons Attribution Non-Commercial License (http://creativecommons.org/licenses/by-nc/4.0) which permits unrestricted non-commercial use, distribution, and reproduction in any medium, provided the original work is properly cited.
} 
shorter screening intervals). ${ }^{5,9}$ The surveillance is conducted every 6 months with ultrasonography (US) and $\alpha$-fetoprotein (AFP) screening for the early detection of HCC in high-risk patients, such as those with cirrhosis or chronic hepatitis $\mathrm{C}$ or $\mathrm{B}$ in Japan. ${ }^{10}$ In Korea, the national surveillance program for LC was established in 2003. In this program, repeated applications of diagnostic tests (AFP and US) at 6-month intervals were recommended in patients at high risk for developing HCC, such as men and women older than 40 years of age with positive hepatitis B surface antigen, anti-hepatitis C virus antibody, or underlying liver cirrhosis. ${ }^{11-13}$

Only a few reports are available to confirm the effectiveness of the national surveillance program. In Japan, the use of active and successful screening and surveillance programs have resulted in a higher proportion of tumors identified at early stages when curative treatments can be applied..$^{5}$ According to followup data from the Nationwide Registry implemented by the Liver Cancer Study Group of Japan, the nationwide 5-year survival rate of HCC patients in Japan was 43\% and 11\% to 15\% in the United States. ${ }^{10}$ There is a general consensus among clinicians that LC surveillance in high-risk groups has the potential to significantly reduce mortality. ${ }^{14}$ However, the results of those studies did not adjust the lead time bias, and no studies were available to evaluate the survival benefit of the National Liver Cancer Surveillance Program (NLCSP) in Korea since the program has started.

This study aimed to investigate the effectiveness of NLCSP in South Korea after the adjustments of various confounding factors in the perspective of survival benefit and cost.

\section{MATERIALS AND METHODS}

\section{Data sources}

This study used the health insurance claims data, LC surveillance data, and qualification data of the National Health Insurance Service (NHIS). The NHIS has comprehensive data sets for diagnoses, treatments including medications and procedures, surgical history, and prescription records of all health-insured or Medicaid patients visiting the hospitals or clinics; as all Korean nationals are covered by the National Health Insurance (NHI) (97\%) or Medicaid (3\%), the NHIS data is fairly representative of the Korean population. The national LC surveillance system requires subjects with liver diseases, such as hepatitis B, hepatitis C, or liver cirrhosis, to undergo annual or biannual medical checkup. The surveillance interval lasted for 6 months from 2003 to 2011 and increased to 1 year from 2012 to 2015. However, in 2016, the surveillance interval was returned to 6 months. The LC surveillance data includes the alanine aminotransferase, hepatitis B surface antigen test, liver US, and AFP results of medically checked subjects under the NLCSP. The qualification data of the beneficiaries includes the information on mortality, income level by insurance type (NHI self-employed subscriber,
NHI employee subscriber, or Medicaid).

This study was approved by the Institutional Review Board of National Evidence-based Healthcare Collaborating Agency (NECA IRB number: 16-003). The informed consent was not required because the data was provided in a de-identified or anonymous form.

\section{Study population}

Individuals diagnosed with liver-related diseases and LC from 2004 to 2015 were included in this study. Liver-related diseases were defined according to the International Classification of Diseases-10th revision (ICD-10) codes (B18, B18.0, B18.1, B18.2, B18.8, B18.9, B19, B19.0, B19.9, K70.1, K70.10, K70.11, K70.2, K70.3, K70.30, K70.31, K70.9, K73, K73.0, K73.1, K73.2, K73.8, K73.9, K74, K74.0, K74.1, K74.2, K74.3, K74.4, K74.5, K74.6, K75.4, K76, K76.1, K765, K76.6, Z22.5). LC was defined with specific cancer identification codes of V193 and C22. Individuals diagnosed with LC from 2006 to 2010 were identified, and the date of first LC diagnosis was defined as the index date. In order to diagnose new cases of LC, the patients who were previously diagnosed with LC or were previously treated for LC 2 years from the index date were excluded. Of the 3,687,656 patients with liver diseases diagnosed from 2006 to 2010 who underwent the NLSCP, 122,975 were diagnosed with LC. In addition, in order to identify patients eligible for the national LC surveillance, we excluded patients less than 40 years of age who have liver-related diseases, have no liver-related diseases, have not undergone the NSCLP prior to the index date, and have a death record before the index date. Among 122,975 patients, 66,632 newly diagnosed LC patients who were eligible target patients for the NSCLP were finally selected as the study population (Fig. 1). The presence and absence of surveillance were determined 2 years prior to the index date. The numbers of patients for the surveillance and no-surveillance groups were $10,527(15.8 \%)$ and 56,105 (84.2\%), respectively. In the surveillance group, the number of surveillance received was further divided into the number of times a particular surveillance was given, that is, $0,1,2$, or more times.

\section{Study design and outcome}

To investigate the survival effects based on the status of surveillance, the subjects were followed from the index date (i.e., in first diagnosis of LC) to death or last observation date (December 31, 2015). Death was defined as all-cause death based on the date of death registered in the NHIS qualification data. If the subjects did not die on the last observation, this subject was censored.

The clinical effects of surveillance were investigated based on the early detection of LC and mortality rate considering the surveillance status. Early detection of LC was defined as patients who were initially treated with hepatectomy, hepatopancreaticoduodenectomy, or radiofrequency ablation (RFA) after diagnosis 
$3,687,656$ Individuals with liver-related disease in 2004 to 2015

122,975 Individuals diagnosed with liver cancer in 2006 to 2010

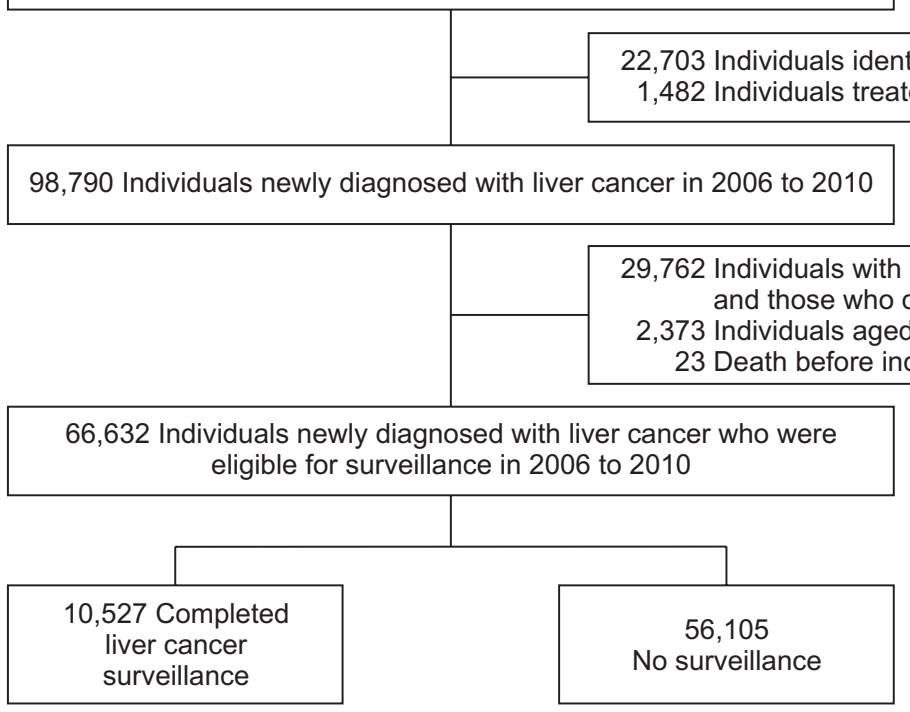

Fig. 1. Flow diagram of the patient selection process.

of LC.

We analyzed changes in medical costs to investigate whether the surveillance program influences the LC medical expense. We extracted claims data incurred for the period from the diagnosis of LC were to death or last observation. For the subjects of each group, total medical costs, such as physician visits, procedures, and prescriptions, incurred during hospitalization and outpatient days. We calculated the monthly medical costs per patient by surveillance status. Monthly costs were calculated on the basis of 30 days per month. All medical costs were converted from South Korean Won (KRW) into 2016 United States Dollars (USD) at a conversion rate of USD $1=\mathrm{KRW} 1,210.5$.

\section{Covariates}

The baseline characteristics such as age, sex, household income level, concomitant diseases and disability were considered as covariates. Household income level was divided into five categories: The household income levels for each employee and self-employed groups were divided into two groups (less than 50\%, over 50\%) and Medicaid beneficiaries. The Medicaid program is managed by the Korean government and is a public assistance scheme to secure the minimum livelihood of lowincome households and to assist with self-help by providing medical services.

In order to determine the health status 1 year prior to the index date, the diagnosis of concomitant diseases and cirrhosis (ICD-10: K74, K74.0-K74.6, K76.1, K70.2, K70.3, K70.30, K70.31) was taken into consideration. We used the Charlson co- morbidity index (CCI) to define the severity of the concomitant diseases. ${ }^{15}$ This index summarizes the information on myocardial infarction, coronary heart disease, peripheral vascular disease, cardiovascular disease, chronic pulmonary disease, rheumatic disease, and renal insufficiency or renal failure.

\section{Lead time bias}

The difference in the time to death between cases diagnosed early and cases diagnosed after symptoms develop exists. Therefore, the survival rate of the surveillance population is overestimated as compared with the no-surveillance population even if the actual surveillance is not effective due to the lead time bias. We used the Schwartz formula ${ }^{16}$ to adjust the lead time bias. In Korea, we used the tumor size in order to calculate the lead time. ${ }^{17}$ The lead times considered in this study were 227, 341, and 455 days for the doubling time 60, 90, and 120 days, respectively. The calculated lead time was considered for the subjects who were positive for surveillance. The length-time bias in direct was adjusted using various tumor doubling times, which may represent tumors with various growth rates, for the calculation of the lead times.

\section{Statistical analysis}

The baseline characteristics of subjects considering their surveillance status were descriptively summarized. To compare the incidence of early LC based on the patient's surveillance status and the number of surveillance, the odds ratio (OR) for the occurrence of LC was calculated using the logistic regression 
model.

In order to compare the mortality difference based on the surveillance status and number of surveillance, the cumulative probability curves derived from the Kaplan-Meier estimates and Cox proportional hazards model were used. The significance of the Kaplan-Meier curve was tested using the Renyi or log-rank test based on the satisfaction of the proportional hazard assumption. The model was also designed to account for the lead time and all confounding variables including sex, age, cirrhosis, disability, CCI, and income level.

To compare the medical costs based on the surveillance status, a generalized linear model (GLM) was used to compute for the total medical costs considering the follow-up. All analyses were performed using the SAS version 9.4 (SAS Institute, Cary, NC, USA), and the proposed p-value was 2-sided with a significance level of 5\%.

\section{RESULTS}

\section{Baseline characteristics}

In the surveillance group, the average age was slightly younger $(60.7 \pm 9.5$ years old vs $61.3 \pm 10.9$ years old), majority of the subjects were males (76.7\% vs $74.9 \%$ ). The surveillance group had more Medicaid patients (16.1\% vs $8.4 \%$ ) and longer median follow-up period (39.8 months vs 18.6 months). In the no surveillance group, the CCI scores were higher $(5.6 \pm 2.4$ vs $6.1 \pm 2.5$ ), whereas the percentages of cirrhosis and disability were lower (63.6\% vs 59.0\% and $19.1 \%$ vs $16.9 \%$ ) (Table 1 ).

\section{Early-stage LC detection rate for patients in surveillance}

After adjustments of the demographic, social, and clinical information, the odds of early-stage LC detection was 1.82 (95\% confidence interval [CI], 1.73 to 1.93) times higher among patients who underwent the NLCSP once in the previous 2-year period than those who did not undergo the surveillance. The rate of early LC detection was 2.58 (95\% CI, 2.27 to 2.94) times higher among patients who underwent NLCSP twice or more in the same period than those who did not undergo the surveillance. In addition, lower income level patients had a lower early LC detection rate. The ORs for Medicaid recipients and NHI employee subscribers with over 50\% income were 0.59 (95\% CI, 0.54 to 0.65 ) and 1.13 (95\% CI, 1.07 to 1.19), respectively, as compared with the reference rate of the NHI self-employed subscriber of $>50 \%$ (Table 2). The surveillance impact on early detection was not different among income groups (Supplementary Table 1).

Table 1. Baseline Characteristics of the Surveillance and No Surveillance Groups of Eligible Patients Newly Diagnosed with Liver Cancer

\begin{tabular}{|c|c|c|}
\hline & Surveillance, no $(\%)(\mathrm{n}=10,527)$ & No surveillance, no. $(\%)(n=56,105)$ \\
\hline \multicolumn{3}{|l|}{ Sex } \\
\hline Male & $8,071(76.7)$ & $41,994(74.9)$ \\
\hline Female & $2,456(23.3)$ & $14,111(25.2)$ \\
\hline Age, mean \pm SD (min, max), yr & $60.7 \pm 9.5(31,93)$ & $61.3 \pm 10.9(33,98)$ \\
\hline$<50$ & $1,381(13.1)$ & $9,029(16.1)$ \\
\hline $50-59$ & $3,398(32.3)$ & $16,778(29.9)$ \\
\hline $60-69$ & $3,769(35.8)$ & $16,395(29.2)$ \\
\hline $70-79$ & $1,761(16.7)$ & $11,158(19.9)$ \\
\hline$\geq 80$ & $218(2.1)$ & $2,745(4.9)$ \\
\hline \multicolumn{3}{|c|}{ Level of income based on type of insurance } \\
\hline Medicaid & $1,695(16.1)$ & $4,702(8.4)$ \\
\hline \multicolumn{3}{|l|}{ NHI self-employed subscriber } \\
\hline$\leq 50 \%$ & $1,241(11.8)$ & $8,495(15.1)$ \\
\hline$>50 \%$ & $1,776(16.9)$ & $11,661(20.8)$ \\
\hline \multicolumn{3}{|l|}{ NHI employee subscriber } \\
\hline$\leq 50 \%$ & $2,657(25.2)$ & $11,697(20.9)$ \\
\hline$>50 \%$ & $3,158(30.0)$ & $19,550(34.9)$ \\
\hline CCI, mean \pm SD (min, $\max )$ & $5.6 \pm 2.4(2,16)$ & $6.1 \pm 2.5(2,21)$ \\
\hline Cirrhosis & 6,696 (63.6) & 33,096 (59.0) \\
\hline Disability & $2,005(19.1)$ & $9,470(16.9)$ \\
\hline Follow-up, median (Q1, Q3), mo & $39.8(9.5,65.1)$ & $18.6(3.9,59.5)$ \\
\hline
\end{tabular}

min, minimum; max, maximum; NHI, National Health Insurance; CCI, Charlson comorbidity index. 
Table 2. Multivariable Logistic Regression for Early Liver Cancer Diagnosis

\begin{tabular}{|c|c|c|}
\hline & Surveillance, OR (95\% CI) & Frequency of surveillance, OR (95\% CI) \\
\hline Surveillance & & - \\
\hline No & Reference & \\
\hline Yes & $1.90(1.80-2.00)$ & \\
\hline Frequency of surveillance & - & \\
\hline 0 & & Reference \\
\hline 1 & & $1.82(1.73-1.93)$ \\
\hline$\geq 2$ & & $2.58(2.27-2.94)$ \\
\hline Sex, male vs female & $0.95(0.90-1.00)$ & $0.95(0.90-1.00)$ \\
\hline \multicolumn{3}{|l|}{ Age, yr } \\
\hline$<50$ & Reference & Reference \\
\hline $50-59$ & $0.98(0.92-1.04)$ & $0.98(0.92-1.04)$ \\
\hline $60-69$ & $0.86(0.81-0.91)$ & $0.86(0.81-0.91)$ \\
\hline $70-79$ & $0.51(0.47-0.55)$ & $0.50(0.47-0.55)$ \\
\hline$\geq 80$ & $0.15(0.12-0.19)$ & $0.15(0.12-0.19)$ \\
\hline Cirrhosis, yes vs no & $0.88(0.84-0.92)$ & $0.88(0.84-0.92)$ \\
\hline Disability, yes vs no & $0.83(0.78-0.88)$ & $0.83(0.78-0.88)$ \\
\hline Charlson comorbidity index & $0.88(0.87-0.88)$ & $0.88(0.87-0.88)$ \\
\hline \multicolumn{3}{|l|}{ Income } \\
\hline Medicaid & $0.60(0.55-0.66)$ & $0.59(0.54-0.65)$ \\
\hline NHI self-employed subscriber $(\leq 50 \%)$ & $0.73(0.67-0.78)$ & $0.73(0.67-0.78)$ \\
\hline NHI self-employed subscriber (>50\%) & Reference & Reference \\
\hline NHI employee subscriber $(\leq 50 \%)$ & $0.92(0.87-0.98)$ & $0.92(0.86-0.98)$ \\
\hline NHI employee subscriber (>50\%) & $1.13(1.07-1.20)$ & $1.13(1.07-1.19)$ \\
\hline
\end{tabular}

Results were obtained from logistic regression analysis.

OR, odds ratio; CI, confidence interval; NHI, National Health Insurance.

\section{Risk of mortality for patients in surveillance}

The survival probability of the surveillance group was statistically higher throughout the entire follow-up period ( $\mathrm{p}<0.0001$, Renyi test) as compared with the no-surveillance group. Mortality increased in the no-surveillance (65.8\% vs 75.8\%) group than in the surveillance group during the follow-up periods. Kaplan-Meire curve also showed that survival probability was higher in surveillance group after the adjustment of the lead time bias (Fig. 2).

In the Cox proportional hazards model adjusted for baseline characteristics, hazard ratio (HR) for mortality was significantly lower in the surveillance group as compared with the nonsurveillance group (HR, 0.77; 95\% Cl, 0.75 to 0.79) (Table 3, Supplementary Table 2). After categorization of surveillance frequency for the previous 2 years, patients who received more frequent surveillance showed trends for lower HR for mortality. After adjusting the lead time to 227, 341, and 445 days, the mortality risk of the surveillance group remained decreased (Table 3).

\section{Total medical costs for patients in surveillance}

The total medical costs during the follow-up period after the LC diagnosis were USD 4,683 (standard deviation [SD], 31,090) per patient for the surveillance group and USD 6,814 (SD, 35,909 ) for the non-surveillance group (Supplementary Table 3). The adjusted cost per day based on the GLM was lower in the surveillance group. The cost estimates of the surveillance group based on the GLM was 0.74 (95\% CI, 0.72 to 0.76) times less than those of the non-surveillance group after adjustments of the demographic, social, and clinical information (Table 4).

\section{DISCUSSION}

Due to the lack of concrete evidence regarding the LC surveillance, further studies should be conducted in order to examine the effectiveness of the LC surveillance on the reduction of mortality. Therefore, in the current study, we investigated the survival outcome of LC patients who received the surveillance program from the time it was implemented in South Korea. The mortality risk of patients who underwent the surveillance pro- 
A No lead time adjustment

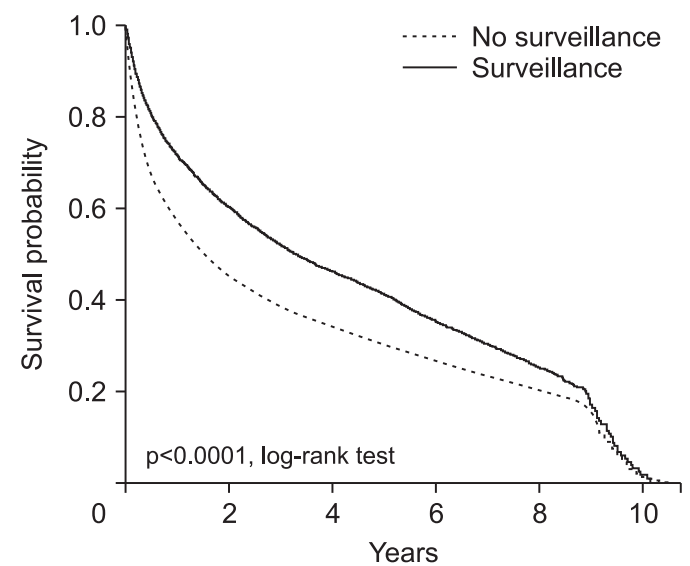

No. at risk

$\begin{array}{ccccccc}\text { No surveillance } & 56,105 & 25,419 & 18,967 & 9,156 & 2,770 & 9 \\ \text { Surveillance } & 10,527 & 6,344 & 4,847 & 1,825 & 421 & 2\end{array}$

C Lead time 341 days

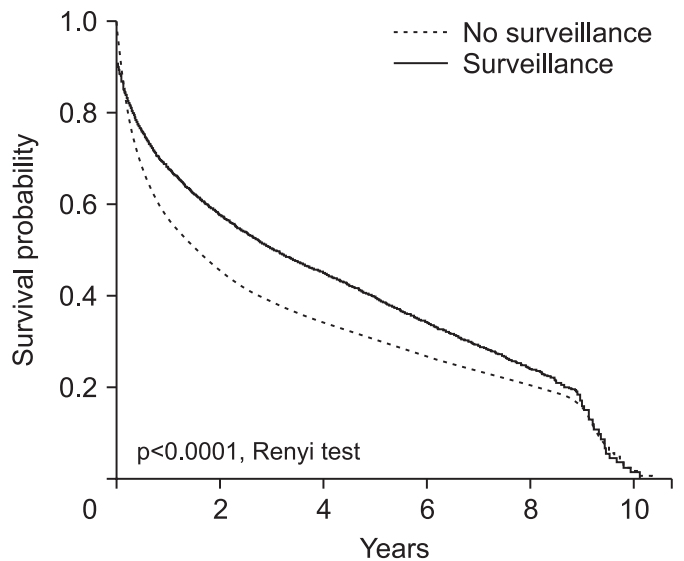

No. at risk

$\begin{array}{lllllll}\text { No surveillance } & 56,105 & 25,419 & 18,967 & 9,156 & 2,770 & 9\end{array}$

B Lead time 227 days

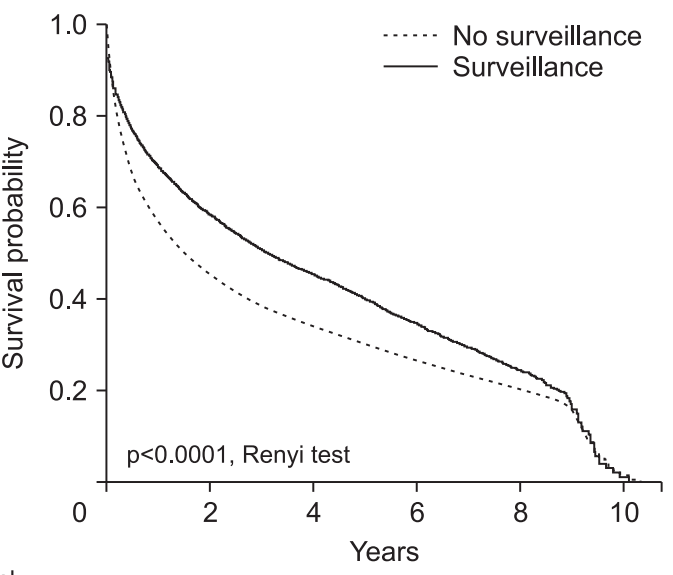

No. at risk

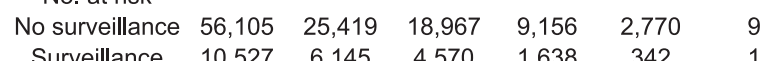

$\begin{array}{lllllll}\text { Surveillance } & 10,527 & 6,145 & 4,570 & 1,638 & 342 & 1\end{array}$

Dead time 445 days

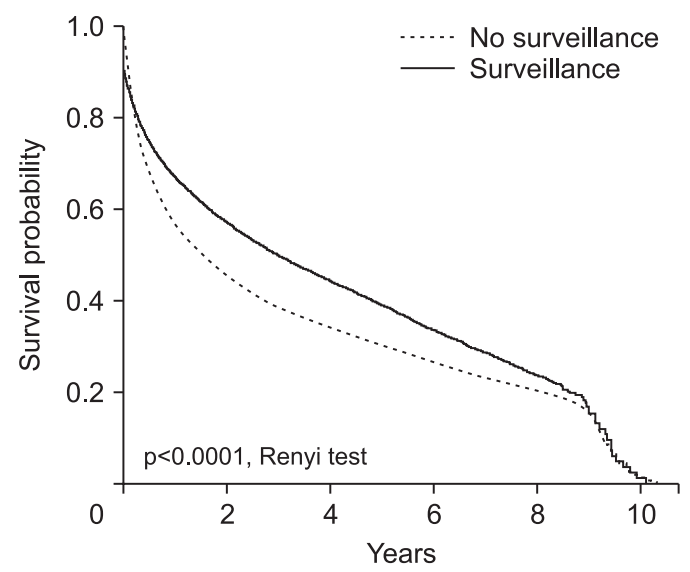

No. at risk

$\begin{array}{lllllll}\text { No surveillance } & 56,105 & 25,419 & 18,967 & 9,156 & 2,770 & 9\end{array}$

$\begin{array}{lllllll}\text { Surveillance } & 10,527 & 5,992 & 4,331 & 1,485 & 297 & 0\end{array}$

Fig. 2. Kaplan-Meier survival curves by surveillance status. (A) Observed cumulative survival with no lead time adjustment. (B) Cumulative survival adjusted with 60 days of median value of tumor doubling time (lead time, 227 days). (C) Cumulative survival adjusted with 90 days of median value of tumor doubling time (lead time, 341 days). (D) Cumulative survival adjusted with 120 days of median value of tumor doubling time (lead time, 445 days).

gram once 2 years prior to the diagnosis of LC was $22.0 \%$ lower (HR, 0.78; 95\% CI, 0.76 to 0.80) as compared with the patients who did not participate in the program. Our result is consistent with the previous results showing the survival benefit of the recipients of surveillance in the national or private setting. HCC patients who underwent surveillance prior to the diagnosis had a smaller tumor size, ${ }^{18,19}$ earlier Barcelona Clinic Liver Cancer tumor stage, ${ }^{20,21}$ received frequent surgical therapy, ${ }^{20}$ and had a better overall survival than those without surveillance. ${ }^{18,20-22}$

The time of survival was calculated from the point of HCC diagnosis. Data were censored at the date of death or last day of follow-up. The lead-time, which is the length of time between detection and death, was adjusted as the survival on the surveillance group can be overestimated due to the early diagnosis of HCC. This lead time bias is one of the potential limitations of various observational studies, and statistical techniques used to adjust the lead time bias were performed in this study as well as in seven earlier observational studies. ${ }^{19,22-27}$ In four studies, ${ }^{22,24-26}$ the Schwartz formula ${ }^{16}$ was used, whereas the remaining studies ${ }^{19,22,23,27}$ used Duffy's approach. ${ }^{28}$ In the study of Wong et al. (2008), ${ }^{26}$ the survival advantage of surveillance disappeared with assumed tumor doubling time of $\geq 120$ days and Tanaka et al. (2006) ${ }^{24}$ reported the survival advantage of surveillance disappeared with 150 days of doubling time. In our study, the survival benefit decreased with doubling time $\geq 120$ days but survival benefit was still significantly higher in surveillance group, and it is consistent results with another Korean study. ${ }^{22}$ Most guidelines (American Association for the Study of Liver Diseases, ${ }^{29}$ European Association for the Study of the Liver, ${ }^{30}$ and Asian Pacific Association for the Study of the Liver ${ }^{12}$ ) rec- 
Table 3. Cox Proportional Hazards Regression for Mortality by Surveillance Status Adjusted for Lead Time Bias among Liver Cancer Patients

\begin{tabular}{|c|c|c|}
\hline & $\begin{array}{c}\text { Crude HR } \\
(95 \% \text { CI) }\end{array}$ & $\begin{array}{c}\text { Adjusted HR } \\
(95 \% \mathrm{CI})^{*}\end{array}$ \\
\hline \multicolumn{3}{|l|}{ No adjustment } \\
\hline Surveillance, yes vs no & $0.73(0.71-0.75)$ & $0.77(0.75-0.79)$ \\
\hline \multicolumn{3}{|l|}{ Frequency of surveillance } \\
\hline 1 vs 0 & $0.74(0.72-0.76)$ & $0.78(0.76-0.80)$ \\
\hline$\geq 2$ vs 0 & $0.68(0.63-0.73)$ & $0.70(0.65-0.76)$ \\
\hline \multicolumn{3}{|l|}{227 Days } \\
\hline Surveillance, yes vs no & $0.79(0.77-0.81)$ & $0.82(0.79-0.84)$ \\
\hline \multicolumn{3}{|l|}{ Frequency of surveillance } \\
\hline 1 vs 0 & $0.78(0.76-0.80)$ & $0.82(0.80-0.84)$ \\
\hline$\geq 2$ vs 0 & $0.73(0.68-0.79)$ & $0.76(0.71-0.82)$ \\
\hline \multicolumn{3}{|l|}{341 Days } \\
\hline Surveillance, yes & $0.79(0.77-0.81)$ & $0.83(0.81-0.86)$ \\
\hline \multicolumn{3}{|l|}{ Frequency of surveillance } \\
\hline 1 & $0.79(0.77-0.82)$ & $0.84(0.82-0.86)$ \\
\hline$\geq 2$ & $0.76(0.7-0.81)$ & $0.79(0.73-0.85)$ \\
\hline \multicolumn{3}{|l|}{445 Days } \\
\hline Surveillance, yes & $0.81(0.79-0.83)$ & $0.85(0.83-0.87)$ \\
\hline \multicolumn{3}{|l|}{ Frequency of surveillance } \\
\hline 1 & $0.81(0.79-0.83)$ & $0.85(0.83-0.88)$ \\
\hline$\geq 2$ & $0.78(0.72-0.84)$ & $0.81(0.76-0.87)$ \\
\hline
\end{tabular}

Results were obtained from Cox proportional hazards regression analysis.

HR, hazard ratio; CI, confidence interval.

*Adjusted for age, sex, cirrhosis, disability, Charlson comorbidity index, level of income.

ommend a 6-month interval in target population, considering that the tumor volume doubling time is approximately 120 days. Based on the available data on HCC tumor doubling times, approximately $40 \%$ of HCC have a tumor doubling time of less than 90 days. ${ }^{27}$ Nevertheless, this is controversial ${ }^{31}$ and further research is needed to evaluate the HCC tumor doubling time, to identify possible factors associated with slow and fast growing tumors, and to define the impact of this information on surveillance strategies.

In this study, Medicaid patients with lower income has slightly lower surveillance rate -which was consistent results with previous study ${ }^{13}$-but has much lower probability of early cancer diagnosis and higher mortality rate than insured payer. Patients receiving regular surveillance were more likely to have higher incomes than those who did not receive surveillance. ${ }^{32}$ Although they have received surveillance, income is significantly associated with disparities in the HCC stage at diagnosis and treatment received. Thus, the disparities of survival outcome evolved from those issues. Follow-up evaluation after an abnormal US and AFP screening requires a diagnostic imaging with contrast-
Table 4. Medical Cost Rates by Surveillance Status among Liver Cancer Patients

\begin{tabular}{lc}
\hline & Cost rate $(95 \% \mathrm{CI})$ \\
\hline Surveillance, yes vs no & $0.74(0.72-0.76)$ \\
Sex, male vs female & $1.18(1.15-1.21)$ \\
Age, yr & Reference \\
$\quad<50$ & $0.96(0.93-0.99)$ \\
$50-59$ & $0.95(0.91-0.98)$ \\
$60-69$ & $1.04(1.00-1.08)$ \\
$70-79$ & $2.00(1.88-2.13)$ \\
$\geq 80$ & $0.97(0.94-0.99)$ \\
Cirrhosis, yes vs no & $1.00(0.97-1.03)$ \\
Disability, yes vs no & $1.26(1.26-1.27)$ \\
Charlson comorbidity index & \\
Income & $1.08(1.03-1.13)$ \\
Medicaid & $1.18(1.13-1.22)$ \\
NHI self-employed subscriber $(\leq 50 \%)$ & Reference \\
NHI self-employed subscriber $(>50 \%)$ & $1.03(0.99-1.07)$ \\
NHI employee subscriber $(\leq 50 \%)$ & $0.92(0.89-0.95)$ \\
NHI employee subscriber $(>50 \%)$ &
\end{tabular}

Results were obtained from generalized linear model analysis. CI, confidence interval; NHI, National Health Insurance.

enhanced computed tomography or magnetic resonance imaging to confirm the diagnosis. The delays in follow-up evaluation are concerning given an approximate tumor doubling time of 90 days for HCC..$^{33}$ Medicaid patients fail to follow up abnormal test results, and surveillance process failures contributed to more advanced tumor stage and poor health outcome. Further studies are needed to assess the impact of delayed or lack of follow-up evaluation on HCC outcomes in the clinical practice.

This study also considered the economic aspect of the surveillance. Even though the total medical costs for the surveillance group were higher, the costs per follow-up day were less as compared with the no-surveillance group. The medical cost for the surveillance group was less compared to the non-surveillance group after adjustments of demographic, social, and clinical information. This may be explained by the fact that longer survival due to the early detection by means of surveillance leads to the higher total costs, but lower costs per follow-up day. The medical cost-effectiveness study to compare the costs and survival benefit would answer the economic benefits of the national surveillance program.

This study has several limitations related to the data source. First, the NHI claims data only includes partial information on the clinical factors. This study aimed to identify whether LC surveillance is effective in diagnosing $\mathrm{LC}$ at an early stage and improving survival outcomes by providing appropriate treatment. However, due to the limitations on NHI claims data, LC patients were defined based on the diagnosis code information available 
in the NHI claims data and the LC stage could not be classified. This may cause over-estimation of the number of patients diagnosed with LC and potentially affect the diagnostic accuracy and early detection. To overcome this problem, early stage LC was defined as LC patients who received treatment such as surgery or RFA that is the mainly used in the localized stages of LC. Furthermore, in this study as this affects both the surveillance and no surveillance groups, this limitation is unlikely to significantly affect the results.

Second, if the patients received a private medical checkup for LC, those procedures were not detected in this study, because we used the NHI claims data. However, the opportunistic cancer screening may not allow much impact on the study results because it may affect either the surveillance or no-surveillance group (Supplementary Table 1). Furthermore, there may be a self-selection bias. People who are voluntarily screened for cancer are more likely to be healthier than those who are not. ${ }^{34}$ However, in this study, the distribution of concomitant diseases, cirrhosis and disability was comparable among the two groups and this result did not support that the possibility of self-selection bias.

Lastly, it is necessary to adjust the lead time bias when analyzing whether the LC surveillance improves the survival rate of LC patients. To adjust for lead time bias, the accurate doubling time of Korean patients with LC is required. However, as mentioned above, this information was not available; to overcome this uncertainty, we conducted a sensitivity analysis by varying the doubling time from 60 to 120 days. Length time bias was not fully considered due to the lack of data. Despite these limitations, this is the first study to analyze the impact of the national LC surveillance program in patient's health and economic outcome. Furthermore, the results are highly generalizable because the study includes all participants and LC patients diagnosed.

In conclusion, this study highlights the survival benefit in patients who underwent the national LC surveillance program and the need for continuous improvements on the national surveillance program in Korea. To meet the purpose of surveillance and to maximize the effect of surveillance, the public health's efforts to encourage the surveillance participation rate for reducing significantly cancer mortality would still be required.

\section{CONFLICTS OF INTEREST}

No potential conflict of interest relevant to this article was reported.

\section{ACKNOWLEDGEMENTS}

This study was supported by National Evidence-based Healthcare Collaborating Agency (grant number: NB16-001). We thank all the professionals from National Health Insurance
Service (NHIS) and all relevant data were obtained from the National Health Insurance Sharing Service (NHIS-2016-1-023).

\section{AUTHOR CONTRIBUTIONS}

Study concept and design: J.W.K., J.H.L., S.S. Data analysis and interpretation: J.W.K., J.K.S. Study supervision: J.W.K., S.S. Drafting of manuscript: H.J.T. Administrative and technical support: H.J.T. Data acquisition, statistical analysis: J.L. Data interpretation: J.H.L. Critical revision of manuscript for important intellectual content: S.S.

\section{ORCID}

Jin Won Kwon

Ha Jin Tchoe

Jayoun Lee

Jae Kyung Suh

Jeong-Hoon Lee

Sangjin Shin

https://orcid.org/0000-0003-3467-7805

https://orcid.org/0000-0001-6830-499X

https://orcid.org/0000-0002-0810-327X

https://orcid.org/0000-0002-9804-2983

https://orcid.org/0000-0002-0315-2080

https://orcid.org/0000-0001-5383-8614

\section{REFERENCES}

1. Ferlay J, Soerjomataram I, Dikshit R, et al. Cancer incidence and mortality worldwide: sources, methods and major patterns in GLOBOCAN 2012. Int J Cancer 2015;136:E359-E386.

2. Jung KW, Won YJ, Oh CM, et al. Cancer statistics in Korea: incidence, mortality, survival, and prevalence in 2014. Cancer Res Treat 2017;49:292-305.

3. Altekruse SF, McGlynn KA, Reichman ME. Hepatocellular carcinoma incidence, mortality, and survival trends in the United States from 1975 to 2005. J Clin Oncol 2009;27:1485-1491.

4. Balogh J, Victor D 3rd, Asham EH, et al. Hepatocellular carcinoma: a review. J Hepatocell Carcinoma 2016;3:41-53.

5. Zhao C, Nguyen MH. Hepatocellular carcinoma screening and surveillance: practice guidelines and real-life practice. J Clin Gastroenterol 2016;50:120-133.

6. Mittal S, Kanwal F, Ying J, et al. Effectiveness of surveillance for hepatocellular carcinoma in clinical practice: a United States cohort. J Hepatol 2016;65:1148-1154.

7. Schweitzer A, Horn J, Mikolajczyk RT, Krause G, Ott JJ. Estimations of worldwide prevalence of chronic hepatitis B virus infection: a systematic review of data published between 1965 and 2013. Lancet 2015;386:1546-1555.

8. Petruzziello A, Marigliano S, Loquercio G, Cozzolino A, Cacciapuoti C. Global epidemiology of hepatitis C virus infection: an up-date of the distribution and circulation of hepatitis $\mathrm{C}$ virus genotypes. World J Gastroenterol 2016;22:7824-7840.

9. Kudo M. Surveillance, diagnosis, treatment, and outcome of liver cancer in Japan. Liver Cancer 2015;4:39-50.

10. Kudo M. Japan's successful model of nationwide hepatocellular carcinoma surveillance highlighting the urgent need for global 
surveillance. Liver Cancer 2012;1:141-143.

11. Yoon SK, Chun HG. Status of hepatocellular carcinoma in South Korea. Chin Clin Oncol 2013;2:39.

12. Omata M, Cheng AL, Kokudo N, et al. Asia-Pacific clinical practice guidelines on the management of hepatocellular carcinoma: a 2017 update. Hepatol Int 2017;11:317-370.

13. Suh M, Song S, Cho HN, et al. Trends in participation rates for the national cancer screening program in Korea, 2002-2012. Cancer Res Treat 2017;49:798-806

14. Lee YH, Choi KS, Jun JK, et al. Cost-effectiveness of liver cancer screening in adults at high risk for liver cancer in the republic of Korea. Cancer Res Treat 2014;46:223-233.

15. Charlson ME, Pompei P, Ales KL, MacKenzie CR. A new method of classifying prognostic comorbidity in longitudinal studies: development and validation. J Chronic Dis 1987;40:373-383.

16. Schwartz M. A biomathematical approach to clinical tumor growth. Cancer 1961;14:1272-1294.

17. Cho EJ, Lee JH, Lee YB, et al. Surveillance for hepatocellular carcinoma may improve patient survival even in patients with decompensated cirrhosis. Proceedings of the 2012 the Korean Association for the Study of the Liver Meeting. 2012 Nov 22; Seoul, Korea.

18. Edenvik P, Davidsdottir L, Oksanen A, Isaksson B, Hultcrantz R, Stål P. Application of hepatocellular carcinoma surveillance in a European setting. What can we learn from clinical practice? Liver Int 2015;35:1862-1871.

19. van Meer S, de Man RA, Coenraad MJ, et al. Surveillance for hepatocellular carcinoma is associated with increased survival: results from a large cohort in the Netherlands. J Hepatol 2015;63:1156-1163.

20. El-Zayadi AR, Badran HM, Shawky S, Emara S, El-Bareedy A, Sobhi M. Effect of surveillance for hepatocellular carcinoma on tumor staging and treatment decisions in Egyptian patients. Hepatol Int 2010;4:500-506.

21. Nusbaum JD, Smirniotopoulos J, Wright HC, et al. The effect of hepatocellular carcinoma surveillance in an urban population with liver cirrhosis. J Clin Gastroenterol 2015;49:e91-e95.

22. Kim HY, Nam JY, Lee JH, et al. Intensity of surveillance for hepatocellular carcinoma determines survival in patients at risk in a hepatitis B-endemic area. Aliment Pharmacol Ther 2018;47:14901501.
23. El-Serag HB, Kramer JR, Chen GJ, Duan Z, Richardson PA, Davila JA. Effectiveness of AFP and ultrasound tests on hepatocellular carcinoma mortality in HCV-infected patients in the USA. Gut 2011;60:992-997.

24. Tanaka H, Nouso K, Kobashi H, et al. Surveillance of hepatocellular carcinoma in patients with hepatitis $\mathrm{C}$ virus infection may improve patient survival. Liver Int 2006;26:543-551.

25. Tong MJ, Sun HE, Hsien C, Lu DS. Surveillance for hepatocellular carcinoma improves survival in Asian-American patients with hepatitis B: results from a community-based clinic. Dig Dis Sci 2010;55:826-835.

26. Wong GL, Wong VW, Tan GM, et al. Surveillance programme for hepatocellular carcinoma improves the survival of patients with chronic viral hepatitis. Liver Int 2008;28:79-87.

27. Cucchetti A, Trevisani F, Pecorelli A, et al. Estimation of lead-time bias and its impact on the outcome of surveillance for the early diagnosis of hepatocellular carcinoma. J Hepatol 2014;61:333341.

28. Duffy SW, Nagtegaal ID, Wallis M, et al. Correcting for lead time and length bias in estimating the effect of screen detection on cancer survival. Am J Epidemiol 2008;168:98-104.

29. Bruix J, Sherman M; American Association for the Study of Liver Diseases. Management of hepatocellular carcinoma: an update. Hepatology 2011;53:1020-1022.

30. European Association for the Study of the Liver; European Organisation for Research and Treatment of Cancer. EASL-EORTC clinical practice guidelines: management of hepatocellular carcinoma. J Hepatol 2012;56:908-943.

31. Sheu JC, Sung JL, Chen DS, et al. Growth rate of asymptomatic hepatocellular carcinoma and its clinical implications. Gastroenterology 1985;89:259-266.

32. Davila JA, Henderson L, Kramer JR, et al. Utilization of surveillance for hepatocellular carcinoma among hepatitis $C$ virus-infected veterans in the United States. Ann Intern Med 2011;154:85-93.

33. Singal AG, Tiro JA, Gupta S. Improving hepatocellular carcinoma screening: applying lessons from colorectal cancer screening. Clin Gastroenterol Hepatol 2013;11:472-477.

34. Otto SJ, Schröder FH, de Koning HJ. Low all-cause mortality in the volunteer-based Rotterdam section of the European randomised study of screening for prostate cancer: self-selection bias? J Med Screen 2004;11:89-92. 